\title{
HEAT KERNEL ON A MANIFOLD WITH \\ A LOCAL HARNACK INEQUALITY
}

\section{ALEXANDER GRIGOR'YAN}

\section{INTRODUCTION}

Let $M$ be a complete non-compact finite dimensional Riemannian manifold and $p(x, y, t)$ be the heat kernel of the corresponding heat equation $u_{t}-\Delta u=0$ associated with the Riemannian metric. In this paper, we are concerned with obtaining heat kernel upper bounds reflecting global geometric properties of the manifold. One of the simplest and the most natural forms of expected estimates reads as follows

$$
p(x, y, t) \leqslant f(t) \exp \left(- \text { const } \frac{r^{2}}{t}\right)
$$

where $r=\operatorname{dist}(x, y)$. For example, in the Euclidean space $\mathbf{R}^{n}$ one can put $f(t)=$ const $\cdot t^{-n / 2}$ while in the hyperbolic space $\mathbf{H}^{n} f(t)=\exp (-$ const $\cdot t)$ for large $t$. There are examples of manifolds for which the heat kernel has an intermediate decay (see [17]).

There are two approaches to a question. The first one is to obtain an estimate covering the widest possible class of manifolds while the second is to try to estimate the heat kernel as sharply as possible, for example, to find the best function $f(t)$, using for this purpose as much information about the manifold as required and, hence, having to consider a more particular variety of manifolds.

An example of the former approach is the statement that for any manifold of bounded geometry the heat kernel decays at least as fast as $1 / \sqrt{t}$. It was first understood by Varopoulos [15], [16], and he proved a little bit weaker statement. The result was independently announced also in the note [6]. 
A complete proof was first given by Chavel and Feldman [2] and for a more general conception of bounded geometry by Coulhon [4]. Both these proofs were based upon a discretization technique (developed in [9], [10], [11], [13] etc.), whose main idea is to replace a manifold by an appropriate graph. From this point of view, the rate $1 / \sqrt{t}$ is not unexpected because this is the magnitude of the heat kernel on the thinnest graph $\mathbf{Z}$.

In this paper, we present a direct proof for manifolds which seems to be more flexible. It is interesting that the proof needs the same geometric hypotheses as discretization arguments of [4] despite the approaches are quite different. We discuss below the class of manifolds which appear as a generalization of the notion of a manifold of bounded geometry.

The second set of results presented in this paper is related to a connection between the heat kernel decay in time variable as $t \rightarrow \infty$ and an isoperimetric property of a manifold. We refer the reader to [8] for the history of this question. In that paper, a theorem was proved which establishes equivalence between the heat kernel on-diagonal estimate

$$
p(x, x, t) \leqslant \frac{\text { const }}{V(c t)}
$$

supposed to be true for all $t>0$ and the isoperimetric inequality of the FaberKrahn type

$$
\lambda_{1}(\Omega) \geqslant \Lambda(\operatorname{Vol} \Omega)
$$

where $\lambda_{1}(\Omega)$ is the first Dirichlet eigenvalue of a pre-compact region $\Omega$ and functions $\Lambda(v)$ and $V(t)$ are expressed each through the other by means of the following transformation

$$
t=\int_{0}^{V(t)} \frac{d v}{v \Lambda(v)}
$$

Whenever we want to restrict our considerations to large values of time only, it becomes natural to take into account only big regions $\Omega$. The theorem cited above does not allow us to do that because for its application we need to control $\Lambda(v)$ for small $v$ so that the integral in (1.4) converges. To avoid having to consider small regions, one should assume a manifold to possess a priori a uniform structure. 
Localization at infinity was done by Chavel and Feldman [2] for manifolds of bounded geometry in the case of a polynomial decay of the heat kernel. They considered a classical (as in Euclidean space) isoperimetric inequality between the area of the boundary and the volume of any region containing a ball of a given radius (they referred to such a situation when dealing with regions containing a fixed-size ball as a modified isoperimetric inequality) and showed that it implies a corresponding heat kernel long time upper estimate.

In this paper, we consider a modified isoperimetric inequality for the first Dirichlet eigenvalue in the spirit of [2], but one which, in addition, covers a superpolynomial scale too. A localization at time infinity becomes possible due to our understanding of a structure of the heat kernel's level sets on a locally Harnack manifold to be defined below.

Let us concentrate now on the notion of bounded geometry, which reflects the fact that a manifold is arranged similarly in a fixed size neighbourhood of any point. There are different definitions of this notion. The following one was introduced in [3].

Definition 1. The manifold $M$ is said to have $C^{k}$ - bounded geometry if an injectivity radius at any point is bounded away from 0 and the covariant derivatives up to the order $k$ of the curvature tensor are bounded from above and below.

The next definition occurs the most frequently (see, for example, [11], [2]).

Definition 2. The manifold $M$ is said to have bounded geometry if an injectivity radius at any point is bounded away from 0 and a Ricci curvature at any point is bounded from below by a (negative) constant.

Finally, a definition of weak bounded geometry was applied in [5].

Definition 3. The manifold $M$ is said to have weak bounded geometry if there is a positive radius $\rho$ such that any geodesic ball of this radius is uniformly quasi-isometric to a Euclidean ball which means that there exists a diffeomorphism of any geodesic ball onto a Euclidean one changing the metric at most in $C$ times, the constant $C$ not depending on the ball. 
Each of these definitions covers a wider class of manifolds than the preceding one. The Varopoulos's conjecture is proved in [2] for manifolds of bounded geometry in the sense of Definition 2. The proof of [4], as well as that of the present paper, covers a class of manifolds which is even wider than that of Definition 3. But first, we introduce a notion of locally Harnack manifolds .

DEFINITION 4. The manifold $M$ is said to be locally Harnack manifold if there is a positive radius $\rho>0$ (which will be referred to as Harnack radius ) such that for any point $x \in M$ the following is true

(a) for any positive numbers $r<R<\rho$

$$
\frac{\operatorname{Vol} B_{R}^{x}}{\operatorname{Vol} B_{r}^{x}} \leqslant a\left(\frac{R}{r}\right)^{n}
$$

(b) Poincaré inequality: for any smooth function $f(x)$ in the ball $B_{R}^{x}$ of a radius $R<\rho$ the following inequality is valid

$$
\int_{B_{R}^{x}}|\nabla f|^{2} \geqslant \frac{b}{R^{2}} \int_{B_{R / 2}^{x}}(f-\bar{f})^{2}
$$

provided

$$
\bar{f} \equiv \frac{1}{\operatorname{Vol} B_{R / 2}^{x}} \int_{B_{R / 2}^{x}} f
$$

where $a, b, n$ are positive constants ( $n$ is normally but not necessarily the dimension of $M$ ).

Let us explain why we apply the name "locally Harnack manifold" in connection with properties (a), (b). The cause is that (a) and (b) are equivalent to the Harnack inequality for the heat equation in any cylinder $B_{R}^{x} \times\left(0, R^{2}\right)$ where $R<\rho$ (see [14] and also [7]).

The conditions (a) and (b) are valid, for example, whenever the manifold has Ricci curvature bounded from below by some (negative) constant $-K$ (see [1]). On the other hand, there are manifolds of constant negative curvature (being therefore locally Harnack manifolds), for example, those of finite volume, which may in no case be regarded as manifolds with a locally uniform geometry. To avoid such situations we have to assume some lower bound of the volume of a geodesic ball to be valid. 
Theorem 1.1. Suppose that $M$ is locally Harnack manifold and the following hypothesis holds for any $x \in M$

$$
\text { Vol } B_{\rho}^{x} \geqslant v_{0} r^{-\alpha}
$$

where $r=\operatorname{dist}(x, y), y$ being a fixed point on $M$ and $v_{0}>0,1>\alpha \geqslant 0$, then for any $D>4, t>\rho^{2}, x \in M$

$$
p(x, y, t) \leqslant \frac{\text { const }}{\sqrt{t^{1-\alpha}}} \exp \left(-\frac{r^{2}}{D t}\right)
$$

where const depends on $a, b, v_{0}, n, \alpha, \rho, D$.

Let us put, for example, $\alpha=0$ i.e. we have independently of the point $y$ that

(c) for any $x \in M \quad \mathrm{Vol} B_{\rho}^{x} \geqslant v_{0}$ Then by Theorem 1.1 for all $x, y \in M$ and for all $t>\rho^{2}$

$$
p(x, y, t) \leqslant \frac{\text { const }}{\sqrt{t}} \exp \left(-\frac{r^{2}}{D t}\right)
$$

A manifold satisfying the conditions (a), (b), (c) may be considered as a natural generalization of a notion "manifold of bounded geometry".

As far as behaviour of the heat kernel for small $t$ is concerned, the following general estimate is a consequence of results of [8] and [7].

Proposition 1.1. If $M$ is locally Harnack manifold with property (c) then the heat kernel satisfies for all $x, y \in M$ the inequality

$$
p(x, y, t) \leqslant \text { const }\left\{\begin{array}{l}
\exp \left(-\lambda_{1}(M) t-\frac{r^{2}}{D t}\right), t \geqslant \rho^{2} \\
t^{-n / 2} \exp \left(-\frac{r^{2}}{D t}\right), t \leqslant \rho^{2}
\end{array}\right.
$$

where $\lambda_{1}(M)$ is the bottom of the spectrum of the operator $-\Delta$ in $L^{2}(M)$, $D>4$ is arbitrary and const depends upon $a, b, v_{0}, n, \rho, D$.

This result yields also a sharp long time heat kernel estimate provided the spectral gap $\lambda_{1}(M)$ is positive; but if $\lambda_{1}(M)=0$ then it does not ensure any decay of the heat kernel in contrast to Theorem 1.1.

Another example where Theorem 1.1 is applicable is the following manifold. Let us consider a surface $M$ of revolution around a straightline in $\mathbf{R}^{n+1}$ of a graph of some function $f(\tau)$ defined on $\mathbf{R}$ (=the straightline). Suppose that 
$f$ is smooth so that $M$ is a manifold, and $f(\tau)=|\tau|^{-\beta}, \beta>0$ for large values of $\tau$, then for a fixed $y \in M, \rho>0$ and for any $x \in M$

$$
\text { Vol } B_{\rho}^{x} \geqslant \text { const } \rho r^{-n \beta}
$$

Since the curvature of the surface in question is bounded from below this manifold is locally Harnack one. Theorem 1.1 gives us (1.8) for $\alpha=-n \beta$ provided $\beta<\frac{1}{n}$. It is interesting that for this surface a lower bound is valid with the same power of $t$ so that Theorem 1.1 gives in this case a sharp estimate. Note that for $\beta>1 / n$ the manifolds under consideration has a finite volume and, thereby, the heat kernel does not approach to 0 at all.

Finally, we consider a modified isoperimetric inequality on the manifold in question.

Theorem 1.2. Suppose that $M$ is a locally Harnack manifold with the condition (c). Let any region $\Omega$ containing a ball of radius $\rho$ satisfy an isoperimetric inequality

$$
\lambda_{1}(\Omega) \geqslant \Lambda(\Omega)
$$

where $\Lambda(v)$ is a positive continuous decreasing function in $\left(v_{0}, \infty\right)$. Let the function $V(t)$ be defined by means of the following identity

$$
t-t_{0}=\int_{v_{0}}^{V(t)} \frac{d v}{v \Lambda(v)}
$$

where $t_{0}=$ const $_{a, b, n} \rho^{2}>0$, then for $t>t_{0}$ and all $x, y \in M$

$$
p(x, y, t) \leqslant \frac{\text { const }}{V(t / 2)}
$$

Moreover if the function $V(t)$ satisfies some additional conditions (see section 4 for details) then for all $x, y \in M, t>t_{0}, D>4$

$$
p(x, y, t) \leqslant \frac{\text { const }}{V(c t)} \exp \left(-\frac{r^{2}}{D t}\right)
$$

where $r=\operatorname{dist}(x, y)$ and constants const, $c$ depend upon $a, b, n, \rho, v_{0}, D$.

Acknowledgement. The author is very grateful to W. Hansen for his help and hospitality during his staying in Bielefeld University where this work was done. 


\section{A LOWER BOUND FOR THE FIRST DiRichlet EIGENVALUE ON A LOCALLY HARNACK MANIFOLD}

The main purpose of this section is to obtain a lower bound for $\lambda_{1}(\Omega)$ via the volume on a locally Harnack manifold. To understand the idea behind the proof, let us first suppose that $M$ is a manifold of weak bounded geometry in the sense of definition 3 .

Consider a bounded region $\Omega$ with a smooth boundary $\partial \Omega$ and its intersections with different balls of radius $\rho$ which are similar to a Euclidean one. If in some of these balls the set $\Omega$ covers at least a half of its volume then by continuity arguments there exists another ball of radius $\rho$ where $\Omega$ covers approximately a half of its volume. Therefore, the surface $\partial \Omega$ divides the ball into two approximately equal parts and by the isoperimetric property of a partition in the Euclidean ball we have that the measure of $\partial \Omega$ is bounded from below by a positive constant.

Otherwise, $\Omega$ occupies in any ball of radius $\rho$ less than a half of the volume and we can devide $\Omega$ into many small parts each of them lying in some of the balls in question and apply the isoperimetric inequality in any ball once again. Omitting details we shall only note that the final result in this case is that the measure of $\partial \Omega$ is at least as large as const $(\operatorname{Vol} \Omega)^{\frac{n-1}{n}}$ as it takes place in $\mathbf{R}^{n}$. Hence, in either case we have obtained some lower bound for the measure of the boundary via the function of the volume of $\Omega$.

Let us note that the isoperimetric inequality of a partition in a ball is nothing but an $L^{1}$-version of Poincaré inequality (b). If we have instead the normal $L^{2}$ - version, then we cannot hope to estimate the area of the boundary via the volume, but we are able to prove a $L^{2}$ - version of this inequality - namely, a lower bound for the first Dirichlet eigenvalue of a region via its volume.

Theorem 2.1. Suppose that the manifold $M$ is a locally Harnack one, then for any pre-compact region $\Omega \subset M$ the estimate holds

$$
\lambda_{1}(\Omega) \geqslant \frac{\mathrm{const}}{\rho^{2}} \min \left(\left(\frac{V_{0}}{\operatorname{Vol} \Omega}\right)^{2},\left(\frac{V_{0}}{\operatorname{Vol} \Omega}\right)^{2 / n}\right)
$$


where $\rho$ is the Harnack radius,

$$
V_{0}=\inf _{x}\left\{\operatorname{Vol} B_{\rho}^{x} \mid B_{\rho}^{x} \cap \Omega \neq \emptyset\right\}
$$

and const $>0$ depends on constants $a, b, n$ from Definition 4.

Proof. Let us consider a non-zero Lipschitz function $u \geqslant 0$ in $\bar{\Omega}$ such that $\left.u\right|_{\partial \Omega}=0$. It suffices to prove that the ratio

$$
\frac{\int_{\Omega}|\nabla u|^{2}}{\int_{\Omega} u^{2}}
$$

is bounded from below by the expression on the right-hand side of (2.1). To this end, let us consider a family of level sets of the function $u: \Omega_{t} \equiv\{u>t\}$ for any $t>0$ (here $t$ is not a time !) and set Vol $\Omega_{t}=m(t)$. Let us associate to any $t>0$ some $t^{\prime}>t$ such that

$$
\operatorname{Vol}\left(\Omega_{t} \backslash \overline{\Omega_{t^{\prime}}}\right) \leqslant \delta \operatorname{Vol} \Omega_{t}
$$

where $\delta \in(0,1)$ is to be chosen later as a function of $V_{0} / \mathrm{Vol} \Omega$. Our first step is to estimate from below the integral

$$
\int_{\Omega_{t} \backslash \overline{\Omega_{t^{\prime}}}}|\nabla u|^{2}
$$

via the function $m(t)$. For this purpose we shall apply the following lemma proved in [7] (lemma 1.1 from that paper).

Lemma 2.1. If the conditions (a) and (b) of the Definition 4 hold in the ball $B_{r}^{x}$ then for any Lipschitz function $u$ in this ball and for all $t<t^{\prime}$

$$
\int_{\left\{t<u<t^{\prime}\right\}}|\nabla u|^{2} \geqslant \operatorname{const} \frac{\left(t^{\prime}-t\right)^{2} A^{-} A^{+}}{r^{2} \operatorname{Vol} B_{r}^{x}}
$$

where

$$
\begin{aligned}
& A^{-}=\operatorname{Vol}\left(\{u \leqslant t\} \cap B_{r / 2}^{x}\right) \\
& A^{+}=\operatorname{Vol}\left(\left\{u \geqslant t^{\prime}\right\} \cap B_{r / 2}^{x}\right)
\end{aligned}
$$


Next we shall consider two cases.

CASE 1. Suppose that there exists a ball $B_{\rho / 10}^{x}$ in which the set $\Omega_{t}$ occupies at least a half of its volume i.e.

$$
\operatorname{Vol}\left(\{u>t\} \cap B_{\rho / 10}^{x}\right) \geqslant \frac{1}{2} \operatorname{Vol} B_{\rho / 10}^{x}
$$

Then by continuity arguments there exists a point $x$ at which the equality attains in (2.7) (here we have made use of unboundedness of the manifold). We intend now to apply lemma 2.1 in the ball $B_{\rho / 5}^{x}$. First we need to estimate from below the corresponding volumes $A^{-}, A^{+}$. Due to the choice of $x$ we have that $A^{-}=\frac{1}{2} \operatorname{Vol} B_{\rho / 10}^{x}$. Obviously, we have the following inequality for $A^{+}$

$$
\begin{aligned}
A^{+} & \geqslant \frac{1}{2} \operatorname{Vol} B_{\rho / 10}^{x}-\operatorname{Vol}\left(\Omega_{t} \backslash \overline{\Omega_{t^{\prime}}}\right) \\
& \geqslant \frac{1}{2} \operatorname{Vol} B_{\rho / 10}^{x}-\delta \operatorname{Vol} \Omega \geqslant c V_{0}-\delta \operatorname{Vol} \Omega \geqslant \frac{c}{2} V_{0}
\end{aligned}
$$

where $c=\frac{1}{2} a^{-1} 10^{-n}$ and $\delta$ is assumed to satisfy the inequality

$$
\delta \mathrm{Vol} \Omega \leqslant \frac{c}{2} V_{0}
$$

Hence, we get

$$
A^{+} \geqslant \text {const } V_{0}
$$

and by lemma 2.1

$$
\int_{\Omega_{t} \backslash \overline{\Omega_{t^{\prime}}}}|\nabla u|^{2} \geqslant \mathrm{const} \frac{\left(t^{\prime}-t\right)^{2} A^{-} A^{+}}{\rho^{2} \operatorname{Vol} B_{\rho / 5}^{x}} \geqslant \mathrm{const} \frac{\left(t^{\prime}-t\right)^{2}}{\rho^{2}} V_{0}
$$

or taking into account (2.8) and $\operatorname{Vol} \Omega \geqslant \operatorname{Vol} \Omega_{t}$ we get finally

$$
\int_{\Omega_{t} \backslash \overline{\Omega_{t^{\prime}}}}|\nabla u|^{2} \geqslant \operatorname{const} \frac{\left(t^{\prime}-t\right)^{2}}{\rho^{2}} \delta m(t)
$$

CASE 2. Suppose now that in any ball $B_{\rho / 10}^{x}$ of radius $\rho / 10$ the set $\Omega_{t}$ occupies less than $\frac{1}{2}$ of its volume, then for any $x \in \Omega_{t}$ there is a radius $r(x)<\rho / 10$ such that

$$
\mathrm{Vol}\left(B_{r(x)}^{x} \cap \Omega_{t}\right)=\frac{1}{2} \mathrm{Vol} B_{r(x)}^{x}
$$


because for a very small $r$ the ball $B_{r}^{x}$ lies completely in $\Omega_{t}$. The union of balls $B_{2 r(x)}^{x}$ covers $\Omega_{t}$. Hence, by means of Banach process we can select from them at most a countable sequence $\left\{B_{2 r_{i}}^{x_{i}}\right\}$ so that they do not intersect each other while the balls $B_{10 r_{i}}^{x_{i}}$ cover together $\Omega_{t}$. We are going to apply lemma 2.1 in every ball $B_{2 r_{i}}^{x_{i}}$. Let us denote by $A_{i}^{-}, A_{i}^{+}$the corresponding volumes from this lemma. Since $A_{i}^{-}=\frac{1}{2} \mathrm{Vol} B_{r_{i}}^{x_{i}}$ it follows that

$$
\int_{\left\{t<u<t^{\prime}\right\} \cap B_{2 r_{i}}^{x_{i}}}|\nabla u|^{2} \geqslant \text { const } \frac{\left(t^{\prime}-t\right)^{2}}{r_{i}^{2}} A_{i}^{+} .
$$

We have by the condition (a) that

$$
\frac{r_{i}}{\rho} \leqslant\left(\frac{\operatorname{Vol} B_{r_{i}}^{x_{i}}}{\operatorname{Vol} B_{\rho}^{x_{i}}}\right)^{1 / n} \leqslant \text { const }\left(\frac{\operatorname{Vol} \Omega}{V_{0}}\right)^{1 / n}
$$

whence

$$
\int_{\left\{t<u<t^{\prime}\right\} \cap B_{2 r_{i}}^{x_{i}}}|\nabla u|^{2} \geqslant \text { const } \frac{\left(t^{\prime}-t\right)^{2}}{\rho^{2}}\left(\frac{V_{0}}{\operatorname{Vol} \Omega}\right)^{2 / n} A_{i}^{+}
$$

Next, note that $\sum_{i} \operatorname{Vol} B_{10 r_{i}}^{x_{i}}$ is at least as large as the volume $\Omega_{t}$. Hence, according to hypothesis (a) we obtain that

$$
\sum_{i} \operatorname{Vol} B_{r_{i}}^{x_{i}} \geqslant \text { const Vol } \Omega_{t}
$$

Taking into account that

$$
A_{i}^{+\cdot}=\frac{1}{2} \operatorname{Vol} B_{r_{i}}^{x_{i}}-\operatorname{Vol}\left(\left(\Omega_{t} \backslash \overline{\Omega_{t^{\prime}}}\right) \cap B_{r_{i}}^{x_{i}}\right)
$$

and, therefore,

$\sum_{i} A_{i}^{+} \geqslant$const Vol $\Omega_{t}-\operatorname{Vol}\left(\Omega_{t} \backslash \overline{\Omega_{t^{\prime}}}\right) \geqslant \operatorname{const} m(t)-\delta m(t) \geqslant \frac{1}{2} \operatorname{const} m(t)$

(where we have assumed that $\delta \leqslant \frac{1}{2}$ const) we obtain from (2.11)

$$
\int_{\Omega_{t} \backslash \overline{\Omega_{t^{\prime}}}}|\nabla u|^{2} \geqslant \text { const } \frac{\left(t^{\prime}-t\right)^{2}}{\rho^{2}}\left(\frac{V_{0}}{\operatorname{Vol} \Omega}\right)^{2 / n} m(t)
$$

We see that in both cases we can choose $\delta$ as follows

$$
\delta=\text { const } \min \left(2, \frac{V_{0}}{\operatorname{Vol} \Omega}\right)
$$


Let us combine the inequalities (2.10) and (2.12) together. If Vol $\Omega \geqslant \frac{1}{2} V_{0}$ then we have $\delta=$ const $\frac{V_{0}}{V_{0} \Omega}$ and in either case 1,2 we get

$$
\int_{\Omega_{t} \overline{\Omega_{t^{\prime}}}}|\nabla u|^{2} \geqslant \operatorname{const} \frac{\left(t^{\prime}-t\right)^{2}}{\rho^{2}} \min \left(\left(\frac{V_{0}}{\operatorname{Vol} \Omega}\right)^{2 / n}, \frac{V_{0}}{\operatorname{Vol} \Omega}\right) m(t)
$$

Otherwise, if $\mathrm{Vol} \Omega<\frac{1}{2} V_{0}$, then the case 2 takes place and (2.14) is valid again (as follows from (2.12)). Therefore, (2.14) holds always provided $t^{\prime}$ and $t$ satisfy (2.4) and $\delta$ is defined from (2.13).

Now we shall arrange an infinite sequence $0=t_{0}<t_{1}<t_{2}<\ldots$ according to the rule

$$
t_{k+1}=\min \left\{\tau \mid \operatorname{Vol}\{u \geqslant \tau\} \geqslant(1-\delta) m\left(t_{k}\right)\right\}
$$

Obviously we have that

$$
m\left(t_{k+1}\right) \leqslant(1-\delta) m\left(t_{k}\right)
$$

in particular, $m\left(t_{k}\right) \rightarrow 0$ as $k \rightarrow \infty$. It follows from (2.15) that

$$
\operatorname{Vol}\left(\Omega_{t_{k}} \backslash \overline{\Omega_{t_{k+1}}}\right) \leqslant \delta \operatorname{Vol} \Omega_{t_{k}}
$$

so the estimate (2.14) is applicable to $t=t_{k}, t^{\prime}=t_{k+1}$ :

$$
\int_{\Omega_{t_{k}} \backslash \overline{\Omega_{t_{k+1}}}}|\nabla u|^{2} \geqslant \mathrm{const} \frac{\left(t_{k+1}-t_{k}\right)^{2}}{\rho^{2}} \min \left(\left(\frac{V_{0}}{\operatorname{Vol} \Omega}\right)^{2 / n}, \frac{V_{0}}{\operatorname{Vol} \Omega}\right) m\left(t_{k}\right)
$$

Let us sum up all the inequalities (2.16) over all $k=0,1,2, \ldots$ and apply the following lemma (see lemma 1.2 in [7]).

Lemma 2.2. Suppose that $\left\{t_{k}\right\}$ is an increasing sequence of real numbers, $t_{0}=0$ and $m_{k}$ is a decreasing sequence of positive numbers such that $m_{k+1} \leqslant$ $(1-\delta) m_{k}$ for some $\delta \in(0,1)$. Then

$$
\sum_{k=0}^{\infty}\left(t_{k+1}-t_{k}\right)^{2} m_{k} \geqslant \frac{\delta}{12} \sum_{k=0}^{\infty} t_{k+1}^{2}\left(m_{k}-m_{k+1}\right)
$$


Therefore, we obtain

$$
\int_{\Omega}|\nabla u|^{2} \geqslant \frac{\text { const }}{\rho^{2}} \delta \min \left(\left(\frac{V_{0}}{\operatorname{Vol} \Omega}\right)^{2 / n}, \frac{V_{0}}{\operatorname{Vol} \Omega}\right) \sum_{k=0}^{\infty} t_{k+1}^{2}\left(m\left(t_{k}\right)-m\left(t_{k+1}\right)\right)
$$

We are left to substitute here the value of $\delta$ from (2.13) and to observe that $m\left(t_{k}\right) \rightarrow 0$ implies the estimate

$$
\int_{\Omega} u^{2} \leqslant \sum_{k=0}^{\infty} t_{k+1}^{2}\left(m\left(t_{k}\right)-m\left(t_{k+1}\right)\right)
$$

so that

$$
\int_{\Omega}|\nabla u|^{2} \geqslant \frac{\text { const }}{\rho^{2}} \min \left(\left(\frac{V_{0}}{\operatorname{Vol} \Omega}\right)^{2 / n},\left(\frac{V_{0}}{\operatorname{Vol} \Omega}\right)^{2}\right) \int_{\Omega} u^{2}
$$

which was to be proved.

\section{UPPER BOUND FOR THE HEAT KERNEL ON A LOCALLY HARNACK MANIFOLD}

We are going to obtain upper bounds for the heat kernel applying arguments of [8]. Let us introduce the notation

$$
E_{D}(x, t)=\int_{M} p^{2}(x, y, t) \exp \left(\frac{r^{2}}{D t}\right) d y
$$

where $r=\operatorname{dist}(x, y), D>2$. As was proved in [8] for any manifold $M$ and for any $D>2$ the function $E_{D}(x, t)$ is always finite and decreasing in $t$. Moreover, the following estimate always holds

$$
p(x, y, t) \leqslant \exp \left(-\frac{r^{2}}{2 D t}\right) \sqrt{E_{D}\left(x, \frac{t}{2}\right) E_{D}\left(y, \frac{t}{2}\right)}
$$

where $r=\operatorname{dist}(x, y)$ (see proposition 5.1 from the paper cited above). This estimate enables one to obtain a Gaussian pointwise upper bound whenever one has proved an estimate of the following kind :

$$
E_{D}(x, t) \leqslant f(t)
$$

To obtain such an estimate we shall use another result of [8] - a particular case of Theorem 4.2 and Corollary 4.2 from there which reads as follows. 
Proposition 3.1. Suppose that for some (fixed) ball $B_{R}^{x} \subset M$ and for any subdomain $\Omega \subset B_{R}^{x}$ the following isoperimetric inequality is valid

$$
\lambda_{1}(\Omega) \geqslant \Lambda(\operatorname{Vol} \Omega)
$$

where the function $\Lambda(v)$ is as follows

$$
\Lambda(v)=\left\{\begin{array}{l}
A v^{-\alpha}, v<V_{0} \\
B v^{-\beta}, v \geqslant V_{0}
\end{array}\right.
$$

$A, B, \alpha, \beta$ being some positive constants and $V_{0}$ is determined from the condition

$$
A V_{0}^{-\alpha}=B V_{0}^{-\beta}
$$

Let the functions $V(t), \mathcal{R}(t)$ be defined by the identities

$$
t=\int_{0}^{V(t)} \frac{d v}{v \Lambda(v)}
$$

and

$$
\mathcal{R}(t)=\int_{0}^{V(t)} \frac{d v}{v \sqrt{\Lambda(v)}} .
$$

Assume also that $R$ and $t$ are related as following

$$
\mathcal{R}(t) \leqslant c R
$$

where $c=c(\alpha, \beta)$. Then

$$
E_{D}(x, t) \leqslant \frac{\text { const }}{V(t)}
$$

with the constant const depending on $D$ and $\sup _{t} t \Lambda(V(t))$.

Remark. The corresponding assertion in [8] was proved for a more general function $\Lambda$ and the statement reads even more bulky. Some simplification occurs here due to the particular polynomial form of $\Lambda$.

Functions $V(t), \mathcal{R}(t)$ are easily computed and admit the following estimates. Let us set

$$
t_{0}=\frac{1}{\alpha} A^{\frac{\beta}{\alpha-\beta}} B^{\frac{\alpha}{\beta-\alpha}}
$$


(this value is found from the condition $V\left(t_{0}\right)=V_{0}$ ). Then we have for any $t<t_{0}$

$$
V(t)=(A \alpha t)^{1 / \alpha}, \quad \mathcal{R}(t)=2 \sqrt{\frac{t}{\alpha}}
$$

and for $t \geqslant t_{0}$

$$
V(t) \geqslant(B \min (\alpha, \beta) t)^{1 / \beta}, \quad \mathcal{R}(t) \leqslant 2 \sqrt{\frac{t_{0}}{\alpha}}+2 \sqrt{\frac{t}{\beta}}
$$

In particular, we see that $t \Lambda(V(t)) \leqslant$ const $_{\alpha, \beta}$. Therefore, the constant in (3.10) depends only on $D, \alpha, \beta$ and what is important to underline it does not depend on $A, B$ as well as the estimates of $\mathcal{R}(t)$ in (3.12) and (3.13).

The relations (3.12) are obtained by a direct computation from the definition of $V(t), \mathcal{R}(t)$. To explain estimates (3.13) let us note that for $t \geqslant t_{0}$ we get from $(3.7)$

$$
t-t_{0}=\int_{V_{0}}^{V(t)} \frac{d v}{v \Lambda(v)}=\frac{1}{B \beta}\left(V(t)^{\beta}-V_{0}^{\beta}\right)
$$

that implies

$$
V(t)^{\beta / 2}-V_{0}^{\beta / 2} \leqslant \sqrt{B \beta t}
$$

Comparing with

$$
R(t)-R\left(t_{0}\right)=\int_{V_{0}}^{V(t)} \frac{d v}{v \sqrt{\Lambda(v)}}=\frac{2}{\sqrt{B} \beta}\left(V(t)^{\beta / 2}-V_{0}^{\beta / 2}\right)
$$

we see that

$$
R(t)-R\left(t_{0}\right) \leqslant 2 \sqrt{\frac{t}{\beta}}
$$

whence, the estimate (3.13) for $R(t)$ follows. To prove the lower bound of $V(t)$ in (3.13) let us note that according to (3.6), $V\left(t_{0}\right)=V_{0}$ and (3.12) we have $V_{0}^{\beta}=\frac{B}{A} V_{0}^{\alpha}=B \alpha t_{0}$. Hence, it follows from (3.14) that

$$
V(t)^{\beta}=V_{0}^{\beta}+B \beta\left(t-t_{0}\right)=B \alpha t_{0}+B \beta\left(t-t_{0}\right) \geqslant B \min (\alpha, \beta) t
$$

The following theorem ensures an upper estimate for $E_{D}(x, t)$ on a locally Harnack manifold. 
Theorem 3.1. Let $M$ be a locally Harnack manifold with a Harnack radius $\rho$. Let us define a function $v_{0}(x, R)$ as the infimum of volumes of all balls $B_{\rho}^{y}$ having a non-empty intersection with the ball $B_{R}^{x}$, then for $D>2, x \in M, t>$ 0

$$
E_{D}(x, t) \leqslant \frac{\text { const }}{v_{0}(x, \bar{c} \sqrt{t}) \min \left(\frac{\sqrt{t}}{\rho},\left(\frac{\sqrt{t}}{\rho}\right)^{n}\right)}
$$

where const depends on $D$ and on the constants $a, b, n$ from definition $4, \bar{c}=$ $\bar{c}(n)$.

Proof. According to Theorem 2.1 any region $\Omega \in B_{R}^{x}$ satisfies the inequality (3.4) with function $\Lambda$ from (3.5) where $\alpha=2 / n, \beta=2$ and

$$
A=\frac{\text { const }}{\rho^{2}} v_{0}(x, R)^{2 / n}, \quad B=\frac{\text { const }}{\rho^{2}} v_{0}(x, R)^{2}
$$

Calculating $t_{0}$ as it is required for proposition 3.1 we get from (3.11) the following

$$
t_{0}=\text { const } \cdot \rho^{2}
$$

To apply proposition 3.1 we choose for any $t>0$ the corresponding $R$ so that the relation (3.9) is satisfied. Let us show that for $R=\frac{1}{c} \sqrt{8 n t}$ (3.9) is valid. Indeed, if $t<t_{0}$ this is obvious because $\mathcal{R}(t)=\sqrt{2 n t}$. Otherwise, if $t \geqslant t_{0}$ we apply the estimate (3.13) for $\mathcal{R}(t)$ and see that

$$
c R=\sqrt{8 n t} \geqslant \sqrt{2 n t_{0}}+\sqrt{2 t} \geqslant \mathcal{R}(t)
$$

We are left to show that

$$
V(t) \geqslant \operatorname{const} v_{0}(x, \bar{c} \sqrt{t}) \min \left(\frac{\sqrt{t}}{\rho},\left(\frac{t}{\rho^{2}}\right)^{n / 2}\right)
$$

Indeed, for $t<t_{0}$ we get from (3.12) and (3.16)

$$
V(t)=\text { const } \cdot v_{0}(x, R)\left(\frac{t}{\rho^{2}}\right)^{n / 2}
$$

For $t \geqslant t_{0}$ we have in the same way

$$
V(t) \geqslant \text { const } \cdot v_{0}(x, R)\left(\frac{t}{\rho^{2}}\right)^{1 / 2}
$$


Substituting here the value of $R$ and applying finally proposition 3.1 we obtain the desired inequality (3.15).

Corollary 3.1. If $M$ is a locally Harnack manifold with a Harnack radius $\rho$ satisfying to the condition (c) of section 1 (i.e. the volume of any ball of radius $\rho$ is at least as large as $\left.v_{0}>0\right)$ then for all $t>\rho^{2}, x \in M, D>2$

$$
E_{D}(x, t) \leqslant \frac{\text { const } \cdot \rho v_{0}^{-1}}{\sqrt{t}}
$$

where const depends on $a, b, n, D$.

Combining Theorem 3.1 with the relation (3.2) we obtain a heat kernel pointwise estimate.

Corollary 3.2. For a Harnack manifold $M$ the following estimate holds for all $x, y \in M, t>\rho^{2}, D>2$

$$
p(x, y, t) \leqslant \frac{\text { const } \cdot \rho}{\sqrt{t}\left(v_{0}(x, \bar{c} \sqrt{t}) v_{0}(y, \bar{c} \sqrt{t})\right)^{\frac{1}{2}}} \exp \left(-\frac{r^{2}}{2 D t}\right)
$$

In particular, we have under conditions of corollary 3.1

$$
p(x, y, t) \leqslant \frac{\text { const } \cdot \rho v_{0}^{-1}}{\sqrt{t}} \exp \left(-\frac{r^{2}}{2 D t}\right)
$$

In both inequalities the constants const depend on $a, b, n, D ; \bar{c}$ is the same as in Theorem 3.1.

The estimate (3.18) can be transformed to be expressed via another function of volume. Indeed, let us fix some point $z$ and put

$$
w(R)=\inf _{x \in B_{R}^{z}} \operatorname{Vol} B_{\rho}^{x}
$$

Obviously, we have

$$
v_{0}(x, R) \geqslant w(d(x)+R+\rho)
$$

where $d(x)=\operatorname{dist}(x, z)$. Applying the foregoing corollary we obtain for $t>\rho^{2}$ and any $x, y \in M$

(3.21) $p(x, y, t) \leqslant \frac{\text { const } \cdot \rho}{\sqrt{t}(w(d(x)+\hat{c} \sqrt{t}) w(d(y)+\hat{c} \sqrt{t}))^{\frac{1}{2}}} \exp \left(-\frac{r^{2}}{2 D t}\right)$ 
where $\hat{c}=\bar{c}+1$.

For example, if $w(R) \geqslant$ const $\cdot R^{-\gamma}, \gamma>0$ it follows that

$$
p(x, y, t) \leqslant \frac{\text { const } \cdot \rho}{\sqrt{t^{1-\gamma}}}\left(\left(\hat{c}+\frac{d(x)}{\sqrt{t}}\right)\left(\hat{c}+\frac{d(y)}{\sqrt{t}}\right)\right)^{\gamma / 2} \exp \left(-\frac{r^{2}}{2 D t}\right)
$$

In particular, if we put here $y=z$ and note that the polynomial $(\hat{c}+r / \sqrt{t})^{\gamma / 2}$ is majorized by the exponential multiple $\exp \left(\varepsilon \frac{r^{2}}{t}\right)$ with an arbitrarily small $\varepsilon>0$ then we obtain nothing but Theorem 1.1 .

\section{MODIFIED ISOPERIMETRIC INEQUALITY}

The heat kernel estimates obtained in the preceding section are valid for a wide class of locally Harnack manifolds but of course as any general estimate they are not sharp for more particular classes of manifolds. Here we impose an additional restriction that a locally Harnack manifold with the condition (c) satisfies some isoperimetric inequality for large domains and obtain a more precise information about the heat kernel decay in time via the isoperimetric function. As was mentioned in Introduction, the main difficulty lies in the fact that we are not given a priori an isoperimetric inequality for all regions (otherwise we could simply apply [8]).

The key point of our proof is that we are able to show that the level sets of the heat kernel on a locally Harnack manifold are similar to geodesic balls. More precisely, a level set (for a fixed time) either lies in some ball or contains a smaller ball, the radii of the balls being finite proportional to the Harnack radius $\rho$. To prove this we apply locally Harnack inequality. Afterwards, to estimate the heat kernel we can repeat arguments of [8] because as turned out they require the isoperimetric inequality only for the level sets of the heat kernel rather than for all regions. We apply for large level sets a given isoperimetric inequality and for small level sets - the isoperimetric inequality inside a small ball (in fact, Theorem 2.1).

Theorem 4.1. Let $M$ be a locally Harnack manifold with a Harnack radius $\rho$, then for any positive $\varepsilon<\varepsilon_{0}=\varepsilon_{0}(a, b, n)$ there exists $\delta=\delta(\varepsilon, a, b, n)>0$ such that for all $x \in M, 0<t<\delta \rho^{2}$ the level set

$$
G_{t}=\{y \in M \mid p(x, y, t)>\varepsilon p(x, x, 2 t)\}
$$


lies in the ball $B_{c_{1} \rho}^{x}$ while for $t \geqslant \delta \rho^{2}$ the set $G_{t}$ contains the ball $B_{c_{2} \rho}^{x}$, where $0<c_{2}<c_{1}<1$ and $c_{1,2}$ depend only on constants $a, b, n$ from the conditions (a), (b).

Proof. The proof will be split onto three steps.

STEP 1. Let us first prove that if $t<\rho^{2}$ and $r=\operatorname{dist}(x, y)<\rho$ then

$$
p(x, y, t) \leqslant \frac{\text { const }_{a, b, n}}{\operatorname{Vol} B_{\sqrt{t}}^{x}} \exp \left(-\frac{r^{2}}{5 t}\right)
$$

Let us take some $x \in M, R<\rho$ and notice that for any region $\Omega \subset B_{R}^{x}$ we have according to Theorem 2.1

$$
\lambda_{1}(\Omega) \geqslant \frac{\text { const }_{a, b, n}}{R^{2}}\left(\frac{\operatorname{Vol} B_{R}^{x}}{\operatorname{Vol} \Omega}\right)^{2 / n}
$$

To explain this first, note that the value of $V_{0}$ defined from (2.2) is finite proportional to $\operatorname{Vol} B_{R}^{x}$ so that we may replace it by $\operatorname{Vol} B_{R}^{x}$. Second, the estimate (2.1) of Theorem 2.1 includes one more term:

$$
\left(\frac{V_{0}}{\operatorname{Vol} \Omega}\right)^{2}
$$

but in the case under consideration it can be omitted for

$$
\operatorname{Vol} \Omega \leqslant \operatorname{Vol} B_{R}^{x}=\text { const }_{a, n} V_{0}
$$

Next, we apply proposition 3.1 in the ball $B_{R}^{x}$ and due to (4.4) we have for $t \leqslant$ const $_{n} R^{2}, D>2$ that

$$
E_{D}(x, t) \leqslant \frac{\operatorname{const}_{D, a, b, n} R^{n}}{t^{n / 2} \operatorname{Vol} B_{R}^{x}}
$$

Since $E_{D}(x, t)$ is decreasing in $t$ it follows that the following estimate holds for all $t>0$

$$
E_{D}(x, t) \leqslant \frac{\text { const }_{D, a, b, n} R^{n}}{\min \left(t, R^{2}\right)^{n / 2} \operatorname{Vol} B_{R}^{x}}
$$

Applying the estimate (3.2) we get that for all $x, y \in M, t>0, R<\rho$

$$
p(x, y, t) \leqslant \frac{R^{n} \operatorname{const}_{D, a, b, n} \exp \left(-\frac{r^{2}}{2 D t}\right)}{\min \left(t, R^{2}\right)^{n / 2}\left(\operatorname{Vol} B_{R}^{x} \operatorname{Vol} B_{R}^{y}\right)^{\frac{1}{2}}}
$$


where $r=\operatorname{dist}(x, y)$. If now $t<\rho^{2}$ and $\operatorname{dist}(x, y)<\rho$ then we set $D=$ 2.5, $R=\sqrt{t}$ and apply the property (a) in a suitable way that yields us that the volumes of balls $B_{R}^{x}, B_{R}^{y}$ are finite proportional whence (4.2) follows.

STEP 2. Let us proof that for $t<\rho^{2}$

$$
p(x, x, 2 t) \geqslant \frac{\text { const }_{a, b, n}}{\operatorname{Vol} B_{\sqrt{t}}^{x}}
$$

Indeed, let us consider the function $u(y, \tau)=\int_{B_{\sqrt{t} / 4}^{x}} p(y, \xi, \tau) d \xi$ (where $t<\rho^{2}$ is fixed) which satisfies the heat equation and to the initial value $u(y, 0)=1$ if $y \in B_{\sqrt{t} / 4}^{x}$. Hence, we can extend this function by 1 for $\tau<0, y \in B_{\sqrt{t} / 4}^{x}$ so that the extended function satisfies the heat equation in $B_{\sqrt{t} / 4}^{x} \times(-\infty,+\infty)$. Applying the Harnack inequality in the cylinder $B_{\sqrt{t} / 4}^{x} \times(-t, t)$ we obtain

$$
\int_{B_{\sqrt{t} / 4}^{x}} p(x, \xi, t) d \xi=u(x, t) \geqslant \operatorname{const}_{a, b, n}
$$

where const ${ }_{a, b, n}>0$ is the corresponding Harnack constant. Therefore, there exists a point $y \in B_{\sqrt{t} / 4}^{x}$ such that

$$
p(x, y, t) \geqslant \frac{\text { const }_{a, b, n}}{\operatorname{Vol} B_{\sqrt{t}}^{x}}
$$

Applying Harnack inequality once again for the function $p(x, \cdot, \cdot)$ in the cylin$\operatorname{der} B_{\sqrt{t} / 2}^{y} \times(0,2 t)$ we get

$$
p(x, x, 2 t) \geqslant \text { const }_{a, b, n} p(x, y, t)
$$

whence (4.8) follows.

Combining the estimates of steps 1,2 we claim that

$$
\frac{p(x, y, t)}{p(x, x, 2 t)} \leqslant \text { const }_{a, b, n} \exp \left(-\frac{r^{2}}{5 t}\right)
$$

provided $y \in B_{\rho}^{x}, t<\rho^{2}$.

Let $t<\delta \rho^{2}$ and $y \notin B_{c_{1} \rho}^{x}$ where constants $\delta, c_{1}$ are to be chosen later. Then (4.10) implies that

$$
\frac{p(x, y, t)}{p(x, x, 2 t)} \leqslant \text { const }_{a, b, n} \exp \left(-\frac{c_{1}^{2}}{5 \delta}\right)
$$


If the right-hand side of (4.11) is less than $\varepsilon$ then any point $y$ under consideration does not land at $G_{t}$ that means that $G_{t}$ lies in the ball $B_{c_{1} \rho}^{x}$. Thus, the first condition to be satisfied by the choice of $c_{1}, \delta$ is the following

$$
\frac{c_{1}^{2}}{\delta}>\operatorname{const}_{a, b, n}\left(1+\log \frac{1}{\varepsilon}\right)
$$

STEP 3. It is standard that Harnack inequality implies the following estimate for any positive solution $u(y, t)$ to the heat equation in $B_{\rho}^{x} \times(0,+\infty)$ : there exists $\tau<t$ such that

$$
u(x, \tau) \leqslant \exp \left(\text { const }_{a, b, n}\left(1+\frac{r^{2}}{t}\right)\right) u(y, t)
$$

provided $r=\operatorname{dist}(x, y)<\frac{1}{2} \rho$ (see [12]). Indeed, let $m>2$ be an integer such that

$$
\frac{r^{2}}{m}<\frac{t}{4}
$$

In particular, $m$ can be taken to satisfy also the inequality

$$
m<4\left(1+\frac{r^{2}}{t}\right)
$$

Let us divide a shortest geodesics connected points $x, y$ into $2 m$ equal parts denoting the corresponding points as $z_{k}, k=0,1, \ldots 2 m$ where $z_{0}=y, z_{2 m}=x$ and consider a sequence of times $t_{k}=t-k\left(\frac{r}{m}\right)^{2}$. Let us apply Harnack inequality in any cylinder

$$
B_{\rho / m}^{z_{k}} \times\left(t_{k}, t_{k}-2\left(\frac{r}{m}\right)^{2}\right)
$$

(note, that $t_{k}-2\left(\frac{r}{m}\right)^{2}>0$ due to $(4.14)$ ) which gives us

$$
u\left(z_{k+1}, t_{k+1}\right) \leqslant \operatorname{const}_{a, b, n} u\left(z_{k}, t_{k}\right) .
$$

By induction we obtain

$$
u\left(x, t_{2 m}\right) \leqslant\left(\text { const }_{a, b, n}\right)^{2 m} u(y, t)
$$

whence (4.13) follows.

Applying (4.13) for $u(y, t)=p(x, y, t)$ and noting that $p(x, x, 2 t) \leqslant p(x, x, \tau)$ (which simply means that $p(x, x, \cdot)$ is a decreasing function) we have

$$
p(x, x, 2 t) \leqslant \exp \left(\operatorname{const}_{a, b, n}\left(1+\frac{r^{2}}{t}\right)\right) p(x, y, t)
$$


or

$$
\frac{p(x, y, t)}{p(x, x, 2 t)} \geqslant \exp \left(- \text { const }_{a, b, n}\left(1+\frac{r^{2}}{t}\right)\right)
$$

Suppose now that $t>\delta \rho^{2}$ and $r<c_{2} \rho$. Then (4.14) implies

$$
\frac{p(x, y, t)}{p(x, x, 2 t)} \geqslant \exp \left(- \text { const }_{a, b, n}\left(1+\frac{c_{2}^{2}}{\delta}\right)\right)
$$

Thus, if the right-hand side of this inequality is greater than $\varepsilon$ i.e.

$$
1+\frac{c_{2}^{2}}{\delta}<\operatorname{const}_{a, b, n} \log \frac{1}{\varepsilon}
$$

then the entire ball $B_{c_{2} \rho}^{x}$ lies in $G_{t}$.

We are left to compare the relations (4.16) and (4.12) to show that they can be satisfied simultaneously. Indeed, we set, for example, $c_{1}=\frac{1}{2}$ and find $\delta$ so that $(4.12)$ is true:

$$
\delta=\frac{\operatorname{const}_{a, b, n}}{1+\log \frac{1}{\varepsilon}}
$$

Substituting this value into (4.16) we obtain the following inequality to be satisfied by choosing of $c_{2}$

$$
c_{2}^{2} \leqslant \operatorname{const}_{a, b, n} \frac{\text { const }_{a, b, n} \log \frac{1}{\varepsilon}-1}{1+\log \frac{1}{\varepsilon}}
$$

Obviously, for sufficiently small $\varepsilon<\varepsilon_{0}(a, b, n)$ the corresponding value of $c_{2}$ exists and does not depend on $\varepsilon$.

Now we can prove the main result of this section - a heat kernel estimate under an isoperimetric inequality supposed to be valid only for large sets.

Theorem 4.2. labelthm4.2 Let $M$ be a locally Harnack manifold with the Harnack radius $\rho$ and $x$ be some (fixed) point on $M$. Suppose that for any precompact region $\Omega$ containing the ball $B_{\rho}^{x}$ the following inequality holds

$$
\lambda_{1}(\Omega) \geqslant \Lambda(\operatorname{Vol} \Omega)
$$

$\Lambda$ being a positive continuous decreasing function defined on $\left(\mathrm{Vol} B_{\rho}^{x}, \infty\right)$, then for all $t>t_{0}=\delta \rho^{2}$ where $\delta=\delta(a, b, n)>0$ we have

$$
p(x, x, 2 t) \leqslant \frac{\text { Const }_{a, b, n}}{V(t)}
$$


where the function $V(t)$ is defined from the relation

$$
t-t_{0}=\int_{v_{0}}^{V(t)} \frac{d v}{v \Lambda(v)}
$$

and $v_{0}=\operatorname{Vol} B_{\rho}^{x}$.

Remarks. 1. The estimate (4.19) is more rough than it is expected in view of the Theorem 2.1 from [8]. In fact, one can prove that

$$
p(x, x, 2 t) \leqslant \frac{\mathrm{const}}{V(\gamma t)}
$$

where $\gamma$ may be taken arbitrarily close to 2 , but under the condition that the ratio $\frac{t}{\rho^{2}}$ is large enough. We have preferred to present a less sharp inequality which however is valid for a more definite range of time.

2. In the course of the proof we find a certain value of $\delta=\delta(a, b, n)$ for which the statement of the theorem holds. In fact, the estimate (4.19) remains valid for smaller values of $\delta$, too but in this case the constant const ${ }_{a, b, n}$ has to depend on $\delta$ as well.

Proof of theorem. To find $\delta$ we choose first some positive $\varepsilon<\min \left(\frac{1}{4}, \varepsilon_{0}\right)$ where $\varepsilon_{0}$ is the same as in Theorem 4.1 ( $\varepsilon$ will be specified at the end of the proof) and put $\delta=\delta(\varepsilon)$ from the relation (4.17) of Theorem 4.1. Let us consider again a set

$$
G_{t}=\{y \in M \mid p(x, y, t)>\varepsilon p(x, x, 2 t)\}
$$

If $t>t_{0}=\delta \rho^{2}$ then the set $G_{t}$ contains by Theorem 4.1 the ball $B_{c_{2} \rho}^{x}$ where $c_{2}$ is the constant from Theorem 4.1. We want to estimate from below $\lambda_{1}\left(G_{t}\right)$ according to (4.18) but we may apply this inequality only for sets, containing the ball $B_{\rho}^{x}$. This is why we shall consider the union $G_{t} \cup B_{\rho}^{x}$. Due to the monotonicity of the first Dirichlet eigenvalue we have

$$
\lambda_{1}\left(G_{t}\right) \geqslant \lambda_{1}\left(G_{t} \cup B_{\rho}^{x}\right) \geqslant \Lambda\left(\operatorname{Vol}\left(G_{t} \cup B_{\rho}^{x}\right)\right)
$$

Evidently, we can compare the volumes as follows

$$
\operatorname{Vol}\left(G_{t} \cup B_{\rho}^{x}\right) \leqslant \operatorname{Vol} G_{t}+\operatorname{Vol} B_{\rho}^{x} \leqslant \operatorname{Vol} G_{t}+a c_{2}^{n} \operatorname{Vol} B_{c_{2} \rho}^{x} \leqslant C \operatorname{Vol} G_{t}
$$


(where $C=1+a c_{2}^{n}$ ) whence the desired estimate follows:

$$
\lambda_{1}\left(G_{t}\right) \geqslant \Lambda\left(C \operatorname{Vol} G_{t}\right) .
$$

We shall apply (4.21) to obtain an upper bound of the function

$$
I(t)=\int_{M} p(x, y, t)^{2} d y=p(x, x, 2 t)
$$

Note that for any positive numbers $p, \xi$ the following inequality is true

$$
p^{2} \leqslant(p-\xi)_{+}^{2}+2 \xi p
$$

which follows obviously from considering of two cases: $p<\xi$ and $p \geqslant \xi$. Let us set $\xi=\varepsilon p(x, x, 2 t)=\varepsilon I(t)$ and apply this inequality to the heat kernel

$$
\int_{M} p(x, y, t)^{2} d y \leqslant \int_{\{p>\xi\}}(p-\xi)^{2} d y+2 \xi \int_{M} p(x, y, t) d y
$$

or, applying $\int_{M} p(x, y, t) d y \leqslant 1$

$$
\int_{\{p>\xi\}}(p-\xi)^{2} d y \geqslant \int_{M} p^{2} d y-2 \xi
$$

Now we are going to estimate the integral over $G_{t}=\{y \mid p(x, y, t)>\xi\}$ on the left-hand side of (4.22) through the Dirichlet integral of the heat kernel over the same set. To this end, we have to show that the level set $G_{t}$ is bounded for any $t>0$. Indeed, it follows from corollary 3.2 that for a large $r=\operatorname{dist}(x, y)$ the value of $p(x, y, t)$ becomes arbitrarily small so that $G_{t}$ lies in some big ball. Hence, we have that

$$
\int_{\{p>\xi\}}|\nabla p|^{2} d y \geqslant \lambda_{1}\left(G_{t}\right) \int_{\{p>\xi\}}(p-\xi)^{2} d y \geqslant \Lambda(C \operatorname{Vol}\{p>\xi\}) \int_{\{p>\xi\}}(p-\xi)^{2} .
$$

On the other hand

$$
\text { Vol }\{p>\xi\} \leqslant \frac{1}{\xi} .
$$

Combining these inequalities with (4.22) we get

$$
\int_{M}|\nabla p|^{2} d y \geqslant \Lambda\left(C \xi^{-1}\right)\left(\int_{M} p^{2}-2 \xi\right) .
$$

Finally, observing that $I^{\prime}(t)=-2 \int_{M}|\nabla p|^{2}$ and replacing $\xi$ by its value we obtain a differential inequality

$$
I^{\prime}(t) \leqslant-2(1-2 \varepsilon) \Lambda\left(\frac{C}{\varepsilon I(t)}\right) I(t)
$$


which is easily integrated and yields for $t>t_{0}$

$$
\int_{I\left(t_{0}\right)}^{I(t)} \frac{d I}{I \Lambda\left(\frac{C}{\varepsilon I}\right)} \leqslant-2(1-2 \varepsilon) \int_{t_{0}}^{t} d t \leqslant-\left(t-t_{0}\right)
$$

(we have applied that $\varepsilon \leqslant \frac{1}{4}$ ). Changing a variable $v=C /(\varepsilon I)$ we obtain

$$
\int_{\frac{C}{\epsilon I\left(t_{0}\right)}}^{\frac{C}{\epsilon I(t)}} \frac{d v}{v \Lambda(v)} \geqslant t-t_{0}
$$

We are left to relate $\frac{C}{\varepsilon I\left(t_{0}\right)}$ to $v_{0}=\operatorname{Vol} B_{\rho}^{x}$, namely, we should find $\varepsilon$ so that the following is true

$$
v_{0} \leqslant \frac{C}{\varepsilon I\left(t_{0}\right)}
$$

- as soon as this is done one can substitute it into (4.26) and a comparison with (4.20) gives

$$
\frac{C}{\varepsilon I(t)} \geqslant V(t)
$$

and

$$
p(x, x, 2 t)=I(t) \leqslant \frac{C}{\varepsilon V(t)}
$$

which was to be proved.

Returning to (4.27) we shall apply the estimate (4.2) from the proof of Theorem 3.1 (or directly this theorem) which gives in this case

$$
I\left(t_{0}\right)=p\left(x, x, 2 t_{0}\right) \leqslant \frac{\text { const }_{a, b, n}}{\operatorname{Vol} B_{\sqrt{t_{0}}}^{x}} \leqslant \frac{\text { const }_{a, b, n}}{\delta^{n / 2} v_{0}}
$$

Comparing this with (4.27) we see that (4.27) is satisfied provided

$$
\delta^{n / 2} \geqslant \text { const }_{a, b, n} \varepsilon
$$

For sufficiently small $\varepsilon$ this is true due to the fact that $\delta$ is a rational function of $\log \frac{1}{\varepsilon}-$ see (4.17).

Corollary 4.1. Suppose that $M$ is a locally Harnack manifold with the condition (c) i.e. the volume of any ball of the Harnack radius $\rho$ is bounded from below by $v_{0}>0$. Let any region $\Omega$ containing a ball of radius $\rho$ satisfy the inequality

$$
\lambda_{1}(\Omega) \geqslant \Lambda(\Omega)
$$


where $\Lambda(v)$ is a positive continuous decreasing function in $\left(v_{0}, \infty\right)$. Let $t_{0}$ be the same as in Theorem 4.2 and the function $V(t)$ is defined by means of (4.20). Suppose that the following additional condition holds:

(4.29) function $t \Lambda(V(t))$ is increasing on $(T,+\infty)$ for some large $T$.

Then for all $x, y \in M, t>t_{0}$ the following estimate holds

$$
p(x, y, t) \leqslant \frac{\text { Const }}{V(c t)} \exp \left(-\frac{r^{2}}{D t}\right)
$$

where $r=\operatorname{dist}(x, y), D>4$ is arbitrary, $c$ and const depend on all constants $a, b, n, v_{0}, \rho, T, D$.

Remark. The condition (4.29) is required to apply a theorem from [8]. It puts some restrictions on a possible behaviour of $V(t)$ as $t \rightarrow \infty$. Let us note that as follows from (4.20) $\Lambda(V(t))=\frac{V^{\prime}(t)}{V(t)}$. Any a more or less regular function $V(t)$ of at least a polynomial growth, for example, $t^{\alpha}, \exp \left(t^{\alpha}\right)$ etc. satisfies (4.29). On the contrary, the function $V(t)=\log t$ does not suit it. We do not lose much with such functions because on the manifold under consideration the heat kernel decreases always at least as fast as $1 / \sqrt{t}$.

Proof. The idea behind the proof is, first, to obtain a heat kernel on-diagonal estimate being valid for all $t>0$, second, to deduce from it an isoperimetric inequality for all bounded domains (including small ones which are not covered by (4.28) ) and, finally, to apply the theorem [8] which ensures a heat kernel bound with a Gaussian term.

Indeed, (4.2) implies for $t<\rho^{2}$ and for all $x$ that

$$
p(x, x, t) \leqslant \frac{1}{C_{1} t^{n / 2}}
$$

while for $t>2 t_{0}=2 \delta \rho^{2}$ we have by Theorem 4.2

$$
p(x, x, t) \leqslant \frac{1}{C_{2} V(t / 2)}
$$

where $C_{1,2}$ depend on $a, b, n, \rho, v_{0}$. Since we can take $\delta$ to be smaller than $\frac{1}{2}$, it follows that for all $x \in M, t>0$ either (4.31) or (4.32) is valid. 
Let us define a new function $\tilde{V} \in C^{1}(0,+\infty)$ so that

$$
\tilde{V}(t)=\left\{\begin{array}{l}
C_{1} t^{n / 2}, t \leqslant 2 t_{0} \\
C_{2} V(t / 2), t \geqslant \rho^{2}
\end{array}\right.
$$

and in the interval $\left(2 t_{0}, \rho^{2}\right)$

$$
\tilde{V}(t) \leqslant \max \left(C_{1} t^{n / 2}, C_{2} V(t / 2)\right)
$$

Hence, for all $t>0$ we have

$$
p(x, x, t) \leqslant \frac{1}{\widetilde{V}(t)}
$$

For the further considerations we need that $\tilde{V}^{\prime}(t) / \widetilde{V}(t)$ is a decreasing function which is certainly true for small arguments as well as for large ones (the latter follows from $V^{\prime}(t) / V(t)=\Lambda(V(t))$ and from monotone decreasing of $\Lambda$ ). For intermediate values of $t$ that can be achieved by a proper choice of the function $\tilde{V}(t)$ - so far it had only to satisfy the inequality (4.34). Moreover, we need also that this function $\widetilde{V}^{\prime}(t) / \widetilde{V}(t)$ has at most polynomial decay that follows from the condition (4.29) for large $t$, from a polynomial form of $\tilde{V}(t)$ for small $t$ and for intermediate values of $t$ can be again obtained by a choice of $\tilde{V}(t)$.

Let us define the function $\tilde{\Lambda}(v)$ by the identity

$$
\tilde{\Lambda}(\tilde{V}(t))=\frac{\tilde{V}^{\prime}(t)}{\tilde{V}(t)}
$$

Obviously, for large $v$ we have

$$
\widetilde{\Lambda}(v)=\frac{1}{2} \Lambda\left(C_{2}^{-1} v\right)
$$

while for small $v \Lambda(v) \sim v^{-2 / n}$. By Theorem 2.2 from [8] the on-diagonal bound (4.35) implies under the conditions on $\tilde{V}(t)$ specified above the following isoperimetric inequality for any bounded region $\Omega$

$$
\lambda_{1}(\Omega) \geqslant \operatorname{const} \tilde{\Lambda}(\mathrm{Vol} \Omega)
$$

On the other hand, this isoperimetric inequality implies by Theorem 5.1 of [8] that for all $x, y \in M, t>0$ a Gaussian upper bound holds

$$
p(x, y, t) \leqslant \frac{\text { const }}{\widetilde{V}(\hat{c} t)} \exp \left(-\frac{r^{2}}{D t}\right)
$$


provided the function $t \widetilde{V}^{\prime}(t) / \widetilde{V}(t)$ is increasing for large $t$ and bounded for small $t$ which is obviously valid in our case. We are left to replace in (4.38) the function $\tilde{V}(t)$ by $C_{2} V(t / 2)$ for $t>\rho^{2}$.

As it is seen from the proof, the modified isoperimetric inequality (4.18) is not only sufficient but necessary condition as well for the upper bound (4.19) to be valid (up to constant multiples). Indeed, as soon as we have got the estimate (4.19) for large $t$ we can combine it with the estimate (4.2) for small $t$ as it has been done in the course of the proof and obtain the isoperimetric inequality (4.37) for all domains which acquires the desired form for large regions.

\section{REFERENCES}

1. Buser, P., A note on the isoperimetric constant, Ann.Scient.Ec.Norm.Sup., 15 (1982), 213-230.

2. Chavel, I. and Feldman, E., Modified isoperimetric constants, and large time heat diffusion in Riemannian manifolds, Duke Math. J., 64 (1991), no.3, 473-499.

3. Cheeger, J., Gromov, M. and Taylor, M., Finite propagation speed, kernel estimates for functions of the Laplace operator, and the geometry of complete Riemannian manifolds, J. Diff. Geom., 17 (1982), 15-53.

4. Coulhon, T., Noyau de chaleur et discretisation d'une variété riemanniene, Israel J. Math., 80 (1992), 289-300.

5. Davies, E.B., Heat kernel bounds, conservation of probability and the Feller property, J. d'Analyse Math., 58 (1992), 99-119.

6. Grigor'yan, A.A., On solutions of the heat equation on non-compact Riemannian manifolds (in Russian), - Uspechi Matem.Nauk., 42 (1987), no.4, 144-145.

7. Grigor'yan, A.A., The heat equation on non-compact Riemannian manifolds (in Russian), Matem. Sbornik, 182 (1991), no.1, 55-87.

Engl. transl. Math.USSR Sb., 72 (1992), no.1, 47-77.

8. Grigor'yan, A.A., Heat kernel upper bounds on a complete non-compact manifold, (1992), to appear in Revista Mathematica Iberioamericana.

9. Gromov, M., Structures métriques pour les Variétés Riemannienes, Paris: Cedic/Ferdnand Nathan, 1981.

10. Kanai, M., Rough isometries, and combinatorial approximations of geometries of noncompact Riemannian manifolds, J. Math. Soc. Japan, 37 (1985), 391-413.

11. Kanai, M., Rough isometries and the parabolicity of manifolds, J. Math. Soc. Japan, 38 (1986), 227-238.

12. Li, P. and Yau, S.-T., On the parabolic kernel of the Schrödinger operator, Acta Math., 156 (1986), no.3-4, 153-201.

13. Lyons, T. and Sullivan, D., Function theory, random paths and covering spaces, J. Diff. Geom., 19 (1984), 299-323.

14. Saloff-Coste, L., A note on Poincaré, Sobolev and Harnack inequality, Duke J. Math., 65 (1992), 27-38. 
15. Varopoulos, N.Th., Brownian motion and random walks on manifolds, Ann. Inst. Fourier (Grenoble), 34 (1984), 243-269.

16. Varopoulos, N.Th., Random walks and Brownian motion on manifolds, Sympos. Math., 29 (1986), 97-109.

17. Varopoulos, N.Th., Groups of superpolynomial growth, Proceedings of the ICM satellite conference on Harmonic analysis, Springer, 1991.

Bielefeld University, Bielefeld, Germany.

E-mail address: grigor@mathematik.uni-bielefeld.de

Received August 2, 1993. 